\title{
Follow-up of high energy neutrinos detected by the ANTARES telescope
}

\author{
Aurore Mathieu*, on behalf of the ANTARES, TAROT, ROTSE, MASTER and SWIFT \\ Collaborations \\ Aix Marseille Université, CNRS/IN2P3, CPPM UMR 7346, 13288, Marseille, France \\ E-mail: amathieu@cppm.in2p3.fr
}

\begin{abstract}
ANTARES is currently the largest neutrino telescope operating in the Northern Hemisphere, aiming at the detection of high energy neutrinos from astrophysical sources. Such observations would provide important clues about the processes at work in those sources, and possibly contribute to discover the sources of high energy cosmic rays. Transient sources such as gamma-ray bursts (GRBs) and core-collapse supernovae (CCSNe) are promising candidates, and multi-messenger programs offer a unique opportunity to detect these transient sources. In this way, a method based on optical and X-ray follow-ups of high energy neutrino alerts has been developed within the ANTARES Collaboration. This program, denoted as TAToO (Telescopes-ANTARES Target-ofOpportunity), triggers a network of robotic optical telescopes (TAROT, Zadko, MASTER) and the Swift-XRT within a delay of few seconds after the neutrino detection. In this contribution, the analysis of optical and X-ray follow-up observations to search for GRBs and CCSNe is presented.
\end{abstract}

The 34th International Cosmic Ray Conference,

30 July- 6 August, 2015

The Hague, The Netherlands

${ }^{*}$ Speaker. 


\section{Introduction}

High energy neutrinos could be produced in the interaction of charged cosmic rays with matter or radiation surrounding astrophysical sources. Even with the recent detection of extraterrestrial high energy neutrinos by the IceCube experiment [1], no astrophysical neutrino source has yet been discovered. Such a detection would be a direct evidence of hadronic acceleration mechanisms and would therefore provide important information on the origin of very high energy cosmic rays.

High energy neutrinos are thought to be produced in several kinds of astrophysical sources, such as GRBs [2], CCSNe [3] or active galactic nuclei (AGN) [4], in which the acceleration of hadrons may occur. These sources also show a transient behavior covering a large range in the time domain, from seconds for GRBs to weeks for CCSNe or AGN. By combining the information provided by the ANTARES neutrino telescope [5] with information coming from other observatories, the probability of detecting a source is enhanced since the neutrino background is significantly reduced in the time window of the transient event.

Based on this idea, a multi-wavelength follow-up program, TAToO, operates within the ANTARES Collaboration since 2009 [6]. It relies on optical and X-ray follow-ups of selected high energy neutrino events very shortly after their detection. This online search is mostly motivated by models of neutrinos from long duration GRBs and CCSNe. Both are thought to produce a jet, which is highly relativistic in case of long GRBs, but only mildly relativistic in case of choked jet CCSNe. Follow-up observations have the potential to reveal the electromagnetic counterpart of these transient candidate neutrino sources.

\section{Neutrino alerts}

After the selection of up-going events, which largely removes the huge background of atmospheric muons, the ANTARES neutrino sample consists mainly of atmospheric neutrinos. To select the events which might trigger an alert, a fast and robust algorithm is used to reconstruct the data [7]. This algorithm uses an idealized detector geometry and is independent of the dynamical positioning calibration. This reconstruction and subsequent quality selections allow the rate of events to be reduced from few $\mathrm{Hz}$ down to few $\mathrm{mHz}$. The remaining events are then passed to a more precise reconstruction tool which allows the up-going direction of the event to be confirmed and the angular resolution to be improved.

To select neutrino candidates with an increased probability to be of cosmic origin, three online neutrino trigger criteria are currently implemented in the TAToO alert system:

- High energy trigger: the detection of a single high energy neutrino.

- Directional: the detection of a single neutrino for which the direction points toward $\left(<0.5^{\circ}\right)$ a local galaxy $(<20 \mathrm{Mpc})$.

- Doublet trigger: the detection of at least two neutrinos coming from similar directions $\left(<3^{\circ}\right)$ within a predefined time window $(<15 \mathrm{~min})$.

The main performances of these three triggers are described in Table 1. Until now, no doublet trigger has been sent to the network. 
Table 1: Performances of the three alert criteria. The third column corresponds to the fraction of events inside a $2^{\circ} \times 2^{\circ}$ field of view.

\begin{tabular}{ccccc}
\hline \hline Trigger & Angular Resolution (median) & Fraction of events in FoV & Muon contamination & Mean energy $^{\mathrm{a}}$ \\
\hline Doublet & $\leq 0.7^{\circ}$ & & $0 \%$ & $\sim 100 \mathrm{GeV}$ \\
single HE & $0.25-0.3^{\circ}$ & $96 \%$ (GRB), $68 \%(\mathrm{SN})$ & $<0.1 \%$ & $\sim 7 \mathrm{TeV}$ \\
single directional & $0.3-0.4^{\circ}$ & $90 \%$ (GRB), $50 \%(\mathrm{SN})$ & $\sim 2 \%$ & $\sim 1 \mathrm{TeV}$ \\
\hline
\end{tabular}

${ }^{a}$ Neutrino energy weighted assuming the atmospheric muon neutrino spectrum.

The trigger criteria are inspired by the features expected from astrophysical sources and are tuned to comply with the alert rate to send to the telescope network. An agreement between ANTARES and the optical telescope collaborations allows a rate of $\sim 25$ alerts per year to be sent to each optical telescope, while an agreement to send 6 alerts per year to the Swift satellite have been accepted. Due to this reduced rate, a subset of the high energy trigger with more restrictive requirements on the neutrino energy, provides a dedicated trigger for the Swift satellite.

The TAToO alert system is able to send alerts within few seconds ( 3-5 s) after the neutrino detection with an angular resolution better than $0.5^{\circ}$. Since 2009 , around 150 and 7 neutrino alerts have successfully been sent to the optical telescope network and the Swift satellite, respectively.

\section{The optical and X-ray follow-up system}

The network is composed of small robotic optical telescopes such as TAROT [8], Zadko [9] and MASTER [10], and has been extended in June 2013 to the Swift-XRT telescope [11] for X-ray follow-up. TAROT is a network of two identical $0.25 \mathrm{~m}$ telescopes with a field of view (FoV) of $1.86^{\circ} \times 1.86^{\circ}$ located in Calern (France) and La Silla (Chile). These telescopes reach a limiting magnitude of $\sim 18.5$ mag with an exposure time of $180 \mathrm{~s}$. Zadko is a one meter telescope located at the Gingin observatory in Western Australia. It covers a FoV of about 0.15 square degrees and can reach a limiting magnitude 1.4 mag deeper compared to the TAROT telescopes with only 60 $\mathrm{s}$ of exposure. Recently, 5 telescopes from the MASTER network have also joined the TAToO program. These telescopes, located in Russia and in South Africa, consist of 5 pairs of tubes with a diameter of $0.40 \mathrm{~m}$ covering a FoV of up to 8 square degrees for each pair of telescopes. Until the end of 2014, the network also comprises the four optical telescopes ROTSE [12], which have progressively stopped their activity. These $0.45 \mathrm{~m}$ telescopes had a FoV of $1.86^{\circ} \times 1.86^{\circ}$ and a sensitivity of $\sim 18.5 \mathrm{mag}$ with $60 \mathrm{~s}$ of exposure. The wide FoV and the fast response of these telescopes (images can be taken less than $20 \mathrm{~s}$ after the neutrino detection) are well suited to the search for transient sources. For each alert, the optical observation strategy is composed of an early follow-up (within 24 hours after the neutrino detection), to search for fast transient sources such as GRB afterglows, complemented by several observations during the two following months, to detect for example the rising light curves of CCSNe. For TAROT telescopes, 6 images of $180 \mathrm{~s}$ exposure are taken for each observation, while for ROTSE, 30 and 8 images of $60 \mathrm{~s}$ are taken for each observation of the early and long follow-up, respectively.

The Swift satellite with its XRT provides a unique opportunity to observe X-ray counterparts to neutrino triggers. The detection sensitivity of the XRT is $5 \times 10^{-13} \mathrm{erg} \mathrm{cm}^{-2} \mathrm{~s}^{-1}$ in $1 \mathrm{ks}$, and an energy band from 0.3 to $10 \mathrm{keV}$ is covered. Due to the small FoV of the XRT (radius $\sim 0.2^{\circ}$ ) and 
the typical error radius of an ANTARES alert $\left(\sim 0.3-0.4^{\circ}\right)$, each observation of a neutrino trigger is composed of 4 tiles of $2 \mathrm{ks}$ each. This mapping covers about $72 \%$ of the ANTARES PSF for a high energy neutrino. The observation strategy is composed of an automatic response to the neutrino trigger with observations starting as soon as possible. There is an online analysis of the data and in the case where an interesting candidate to be the counterpart is found, further observations are scheduled.

Images provided by follow-up observations must be processed. Optical images are analyzed with a dedicated pipeline based on the image subtraction method ${ }^{1}$, while X-ray data are automatically analyzed by detection algorithms at the UK Swift Science Data Centre.

\section{Results}

\subsection{Early follow-up}

42 neutrino alerts from January 2010 to January 2015 with early optical images $(<24 \mathrm{~h}$ after the neutrino alert) have been analyzed. No optical counterpart associated to one of the 42 neutrinos has been found. Upper limits on the magnitude of possible transient sources which could have emitted the neutrino have thus been derived and are listed in Table 2. These limits correspond to the limiting magnitude of images, which is the faintest signal that can be detected. As we are looking for rapidly-fading sources, the signal is supposed to be more important in the first image of the observation, so the upper limits are the limiting magnitude of each first image computed at $5 \sigma$ and corrected for Galactic extinction [13].

Concerning X-ray follow-up, the Swift-XRT responded to 7 neutrino triggers between mid 2013 and the beginning of 2015. 22 X-ray sources have been found in the tiled analysis and only 2 sources were already catalogued. Although 20 new X-ray sources have been detected, none of them can be clearly associated with the neutrino trigger. Upper limits on the flux density one may expect from an X-ray counterpart have thus been derived (see Table 3). These limits correspond to the sensitivity reached for each 4-tile observation, which lasted from 0.8 to $1.9 \mathrm{ks}$ for the 7 alerts.

\subsection{Discussion on GRB association}

GRBs are the major candidates as sources of high-energy neutrinos among the population of fast transient sources. Because in this study no optical and X-ray counterpart has been observed in coincidence with the 42 and 7 neutrino alerts respectively, the probability to reject a GRB association to each neutrino alert can be directly estimated. To do so, a comparison is done between upper limits obtained for each neutrino alert with optical and X-ray detected afterglow light curves, as shown in Fig. 1.

The cumulative distribution functions (CDFs) of optical afterglow magnitudes and X-ray afterglow fluxes are computed at times coincident with the first optical and X-ray observation of the neutrino alerts, respectively. Figure 2 shows these CDFs at typical times after the GRB in the two wavelengths. Under the hypothesis that each detected neutrino comes from GRB, the probability to reject this hypothesis, $P_{\text {reject }}^{G R B, v}$, can be directly extracted from the CDFs and by considering that the GRB occurred in the field of view of the telescopes. These probabilities are listed in Table 2 and 3

\footnotetext{
${ }^{1}$ The MASTER Collaboration analyzes its images with its own reduction pipeline.
} 

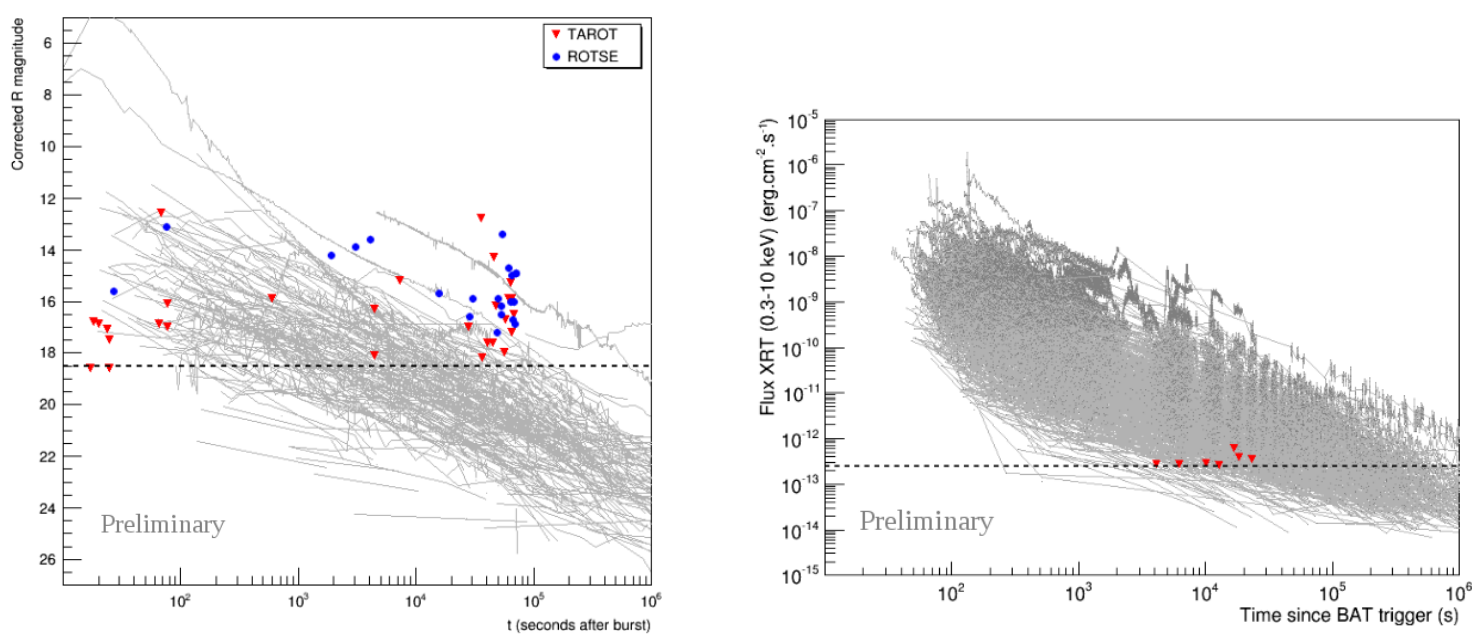

Figure 1: Left: optical afterglow light curves observed from 1997 to 2014 by optical telescopes with upper limits on GRB afterglow magnitude for neutrino alerts followed by TAROT (red triangles) and ROTSE (blue circles). Each point represents the first image of the observation which corresponds to an exposure of $180 \mathrm{~s}$ for TAROT and $20 \mathrm{~s}$ or $60 \mathrm{~s}$ for ROTSE images. The horizontal dashed line corresponds to the sensitivity of these telescopes. Right: 689 X-ray afterglow light curves detected by the Swift-XRT from 2007 to 2015. Upper limits on GRB fluxes for 7 neutrino alerts are represented by red triangles. The horizontal dashed line corresponds to the sensitivity reached with a 2 ks exposure.
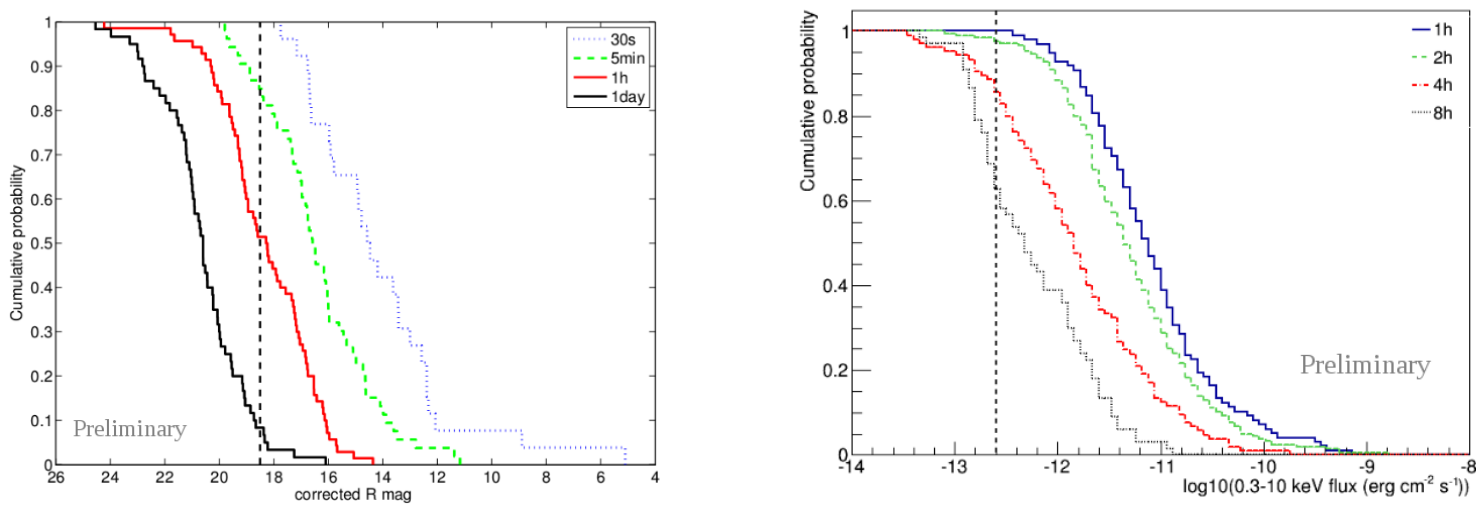

Figure 2: Cumulative distribution of optical (left) and X-ray (right) afterglow magnitudes for 301 and 689 detected GRBs, respectively. Each curve represents different time after burst. The vertical dashed lines represent the sensitivity of the optical telescopes and the XRT.

for the optical and X-ray follow-ups. For most of the really early optical observations $(<1$ min after the neutrino trigger), a GRB association is rejected with $\sim 90 \%$ probability. With X-ray follow-up, a GRB origin of neutrino alerts can be excluded with $\sim 70 \%$ probability for observations made no later than $\sim 2 \mathrm{~h}$ after the trigger.

\subsection{Long term follow-up}

71 alerts from October 2009 to January 2015 with optical follow-up observations (at least three night of observations) have been processed. No slowly varying transient optical counterpart was 
Table 2: Details of the 42 neutrino alerts for which early optical images have been taken.

\begin{tabular}{|c|c|c|c|c|c|c|c|}
\hline Alert name & Telescope & $\begin{array}{l}\text { Analyzed } \\
\text { images }\end{array}$ & $\begin{array}{c}\text { Exposure }^{a} \\
(\mathrm{sec})\end{array}$ & Delay $^{b}$ & $\begin{array}{l}\mathrm{M}_{\text {lim }}{ }^{\mathrm{c}} \\
\text { (mag) }\end{array}$ & $\begin{array}{c}A_{v}^{\mathrm{d}} \\
(\mathrm{mag})\end{array}$ & $\mathrm{P}^{\mathrm{e}}$ \\
\hline ANT100123A & TAROT & 6 & 180 & $17 \mathrm{~h} 47 \mathrm{~m}$ & 15.3 & 0.2 & 0 \\
\hline \multirow[t]{2}{*}{ ANT100725A } & TAROT & 6 & 180 & $1 \mathrm{~m} 17 \mathrm{~s}$ & 16.1 & 0.3 & 0.50 \\
\hline & ROTSE & 30 & 20 & $1 \mathrm{~m} 15 \mathrm{~s}$ & 13.1 & 0.3 & 0.12 \\
\hline ANT100913A & TAROT & 6 & 180 & $11 \mathrm{~h} 24 \mathrm{~m}$ & 17.6 & 0.0 & 0.06 \\
\hline ANT100922A & ROTSE & 26 & 20 & $1 \mathrm{~h} 08 \mathrm{~m}$ & 13.6 & 0.5 & 0 \\
\hline ANT110305A & ROTSE & 29 & 60 & $4 \mathrm{~h} 19 \mathrm{~m}$ & 15.7 & 0.1 & 0.06 \\
\hline ANT110409A & TAROT & 6 & 180 & $1 \mathrm{~m} 08 \mathrm{~s}$ & 12.6 & 5.6 & 0.04 \\
\hline ANT110531A & TAROT & 6 & 180 & $12 \mathrm{~h} 34 \mathrm{~m}$ & 17.6 & 0.1 & 0.06 \\
\hline ANT110923A & TAROT & 7 & 180 & $9 \mathrm{~h} 58 \mathrm{~m}$ & 12.8 & 3.9 & 0 \\
\hline \multirow[t]{2}{*}{ ANT110925B } & TAROT & 6 & 180 & $2 \mathrm{~h} 01 \mathrm{~m}$ & 15.2 & 1.8 & 0.10 \\
\hline & ROTSE & 30 & 60 & $50 \mathrm{~m} 58 \mathrm{~s}$ & 13.9 & 1.8 & 0 \\
\hline ANT111008A & TAROT & 5 & 180 & $12 \mathrm{~h} 53 \mathrm{~m}$ & 14.3 & 2.5 & 0 \\
\hline ANT111019A & ROTSE & 8 & 60 & $18 \mathrm{~h} 22 \mathrm{~m}$ & 16.7 & 0.1 & 0.02 \\
\hline ANT111019B & ROTSE & 8 & 60 & $19 \mathrm{~h} 09 \mathrm{~m}$ & 16.9 & 0.1 & 0.02 \\
\hline ANT111101A & ROTSE & 8 & 60 & $13 \mathrm{~h} 33 \mathrm{~m}$ & 17.2 & 0.1 & 0.02 \\
\hline ANT111205A & TAROT & 6 & 180 & $10 \mathrm{~h} 05 \mathrm{~m}$ & 18.2 & 0.4 & 0.16 \\
\hline \multirow[t]{2}{*}{ ANT111228A } & TAROT & 6 & 180 & $7 \mathrm{~h} 44 \mathrm{~m}$ & 17.0 & 0.1 & 0.04 \\
\hline & ROTSE & 8 & 60 & $7 \mathrm{~h} 53 \mathrm{~m}$ & 16.6 & 0.1 & 0.04 \\
\hline ANT120102A & TAROT & 4 & 180 & $1 \mathrm{~m} 17 \mathrm{~s}$ & 17.0 & 0.1 & 0.60 \\
\hline ANT120105A & ROTSE & 8 & 60 & $17 \mathrm{~h} 39 \mathrm{~m}$ & 16.0 & 0.4 & 0.02 \\
\hline ANT120730A & TAROT & 26 & 180 & $20 \mathrm{~s}$ & 16.9 & 0.4 & 0.88 \\
\hline ANT120907A & TAROT & 14 & 180 & $9 \mathrm{~m} 53 \mathrm{~s}$ & 15.9 & 0.2 & 0.31 \\
\hline \multirow[t]{2}{*}{ ANT120907B } & TAROT & 11 & 180 & $18 \mathrm{~h} 15 \mathrm{~m}$ & 17.2 & 0.2 & 0.02 \\
\hline & ROTSE & 27 & 60 & $8 \mathrm{~h} 28 \mathrm{~m}$ & 15.9 & 0.2 & 0.02 \\
\hline ANT120923A & TAROT & 6 & 180 & $15 \mathrm{~h} 43 \mathrm{~m}$ & 18.0 & 0.1 & 0.03 \\
\hline ANT121010A & TAROT & 24 & 180 & $25 \mathrm{~s}$ & 18.6 & 0.0 & 0.90 \\
\hline ANT121012A & TAROT & 6 & 180 & $19 \mathrm{~h} 06 \mathrm{~m}$ & 16.5 & 0.7 & 0.02 \\
\hline ANT121027A & ROTSE & 8 & 20 & $14 \mathrm{~h} 56 \mathrm{~m}$ & 13.4 & 2.6 & 0 \\
\hline ANT121206A & ROTSE & 27 & 60 & $27 \mathrm{~s}$ & 15.6 & 1.1 & 0.62 \\
\hline ANT130210A & ROTSE & 8 & 60 & $14 \mathrm{~h} 46 \mathrm{~m}$ & 16.5 & 0.1 & 0.02 \\
\hline ANT130724A & TAROT & 3 & 180 & $18 \mathrm{~h} 04 \mathrm{~m}$ & 15.9 & 0.1 & 0.02 \\
\hline ANT130928A & ROTSE & 8 & 60 & $13 \mathrm{~h} 49 \mathrm{~m}$ & 15.9 & 0.1 & 0.02 \\
\hline ANT131027A & ROTSE & 8 & 20 & $18 \mathrm{~h} 14 \mathrm{~m}$ & 15.0 & 0.7 & 0 \\
\hline ANT131209A & TAROT & 6 & 180 & $1 \mathrm{~h} 14 \mathrm{~m}$ & 16.3 & 0.1 & 0.14 \\
\hline ANT131221A & TAROT & 2 & 180 & $18 \mathrm{~s}$ & 16.8 & 0.5 & 0.83 \\
\hline ANT140123A & TAROT & 23 & 180 & $13 \mathrm{~h} 21 \mathrm{~m}$ & 16.2 & 1.3 & 0.02 \\
\hline ANT140125A & TAROT & 6 & 180 & $1 \mathrm{~h} 14 \mathrm{~m}$ & 18.1 & 0.0 & 0.43 \\
\hline ANT140203A & ROTSE & 8 & 60 & $19 \mathrm{~h} 43 \mathrm{~m}$ & 14.9 & 0.1 & 0 \\
\hline \multirow[t]{2}{*}{ ANT140223A } & TAROT & 3 & 180 & $17 \mathrm{~h} 08 \mathrm{~m}$ & 15.9 & 0.1 & 0.02 \\
\hline & ROTSE & 3 & 60 & $31 \mathrm{~m} 29 \mathrm{~s}$ & 14.2 & 0.1 & 0.02 \\
\hline ANT140304A & TAROT & 18 & 180 & $25 \mathrm{~s}$ & 17.5 & 0.6 & 0.92 \\
\hline ANT140309A & TAROT & 16 & 180 & $24 \mathrm{~s}$ & 17.1 & 0.1 & 0.88 \\
\hline ANT140323A & ROTSE & 8 & 60 & $14 \mathrm{~h} 47 \mathrm{~m}$ & 16.2 & 0.2 & 0.02 \\
\hline \multirow[t]{2}{*}{ ANT140408A } & TAROT & 6 & 180 & $16 \mathrm{~h} 11 \mathrm{~m}$ & 16.7 & 0.1 & 0.02 \\
\hline & ROTSE & 8 & 60 & $19 \mathrm{~h} 07 \mathrm{~m}$ & 16.0 & 0.1 & 0.02 \\
\hline ANT140505A & ROTSE & 2 & 60 & $17 \mathrm{~h} 11 \mathrm{~m}$ & 14.7 & 0.1 & 0 \\
\hline ANT140914A & TAROT & 13 & 180 & $1 \mathrm{~m} 05 \mathrm{~s}$ & 16.9 & 0.5 & 0.62 \\
\hline ANT150122A & TAROT & 8 & 180 & $17 \mathrm{~s}$ & 18.6 & 0.1 & 0.90 \\
\hline
\end{tabular}


Table 3: Details of the 7 ANTARES triggers observed by the Swift-XRT since 2013.

\begin{tabular}{|c|c|c|c|c|c|c|c|}
\hline $\begin{array}{c}\text { Trigger name } \\
\text { (ANTyymmddA) }\end{array}$ & $\begin{array}{c}\text { Error radius } \\
\left(^{\circ}\right)\end{array}$ & $\begin{array}{l}\text { Delaya }^{\mathrm{a}} \\
\text { (hours) }\end{array}$ & $\begin{array}{c}\text { Mean exposure } \\
(\mathrm{ks})\end{array}$ & $\begin{array}{c}\text { Sensitivity } \\
\left(\times 10^{-13} \mathrm{erg} \mathrm{cm}^{-2} \mathrm{~s}^{-1}\right)\end{array}$ & New sources (total) ${ }^{b}$ & $\begin{array}{l}\text { Counterpart } \\
\text { candidates }\end{array}$ & $\mathrm{P}^{\mathrm{c}}$ \\
\hline ANT130722A & 0.4 & 1.1 & 1.8 & 2.74 & $4(5)$ & 0 & 0.71 \\
\hline ANT130915A & 0.3 & 6.5 & 1.4 & 3.48 & $2(2)$ & 0 & 0.60 \\
\hline ANT130927A & 0.4 & 5.1 & 1.3 & 3.84 & $0(1)$ & 0 & 0.60 \\
\hline ANT140123A & 0.35 & 4.7 & 0.8 & 5.99 & $1(1)$ & 0 & 0.55 \\
\hline ANT140311A & 0.35 & 2.8 & 1.7 & 2.88 & $3(3)$ & 0 & 0.68 \\
\hline ANT141220A & 0.4 & 3.5 & 1.9 & 2.63 & $4(4)$ & 0 & 0.67 \\
\hline ANT150129A & 0.35 & 1.7 & 1.9 & 2.67 & $6(6)$ & 0 & 0.69 \\
\hline
\end{tabular}

a Delay between the neutrino trigger and the first observation by the Swift-XRT.

b Number of uncatalogued sources among the total number of detected sources in each 4-tile observation.

c Probability to reject an association between the neutrino trigger and a GRB.

found in association with a neutrino trigger. This null result is consistent with the small expectation value of 0.2 accidentally discovered $\mathrm{SNe}$ for 71 alerts.

From this result, constraints on the Ando \& Beacom model [3] parameters will be set. In this model, the production of high energy neutrinos from mildly relativistic jets of CCSNe is proposed, depending on the jet energy $E_{j e t}$, the Lorentz boost factor $\Gamma$ and the rate of CCSNe with such jets $\rho$. To test this model, a test statistic depending on an ANTARES term and an optical follow-up term will be used to check the compatibility of the measurement with the model expectations. If a set of model parameters predicts a significant larger amount of neutrinos and SN counterparts than measured in the data sample, the model can be excluded.

\section{Conclusion}

The optical and X-ray follow-ups of the ANTARES neutrino alerts have been running stably since 2010 and mid 2013, respectively. About 150 and 7 alerts have been sent to the optical telescope network and to the Swift-XRT. The main advantage of the ANTARES program is that it is able to send alerts within few seconds after the neutrino detection with a precision better than $0.5^{\circ}$ for high energy neutrinos. The analysis of 42 and 7 early follow-up observations in optical and X-ray has not yet permitted to discover any transient sources associated to the neutrino events. Upper limits on the magnitude of possible transient sources have been derived. Compared to the state-of-the-art of the GRB afterglow detected light curves, the very rapid response time of the optical telescopes has allowed stringent constraints on the GRB origin of individual neutrinos to be placed. Even with the larger response time of the XRT follow-up, early observations have allowed the GRB origin for the 7 neutrino alerts to be excluded with a high probability.

\section{References}

[1] M. G. Aartsen et al., Science 342 (2013) 1

[2] S. Razzaque, P. Meszaros and E. Waxman, Phys. Rev. Lett. 90 (2003) 241103

[3] S. Ando and J. Beacom, Phys. Rev. Lett. 95 (2005) 061103

[4] A. A. Abdo et al., ApJ 722520 
[5] M. Ageron et al., ANTARES Collaboration, Nucl. Instrum. Meth. A 656 (2011) 11

[6] M. Ageron et al., ANTARES Collaboration, Astropart. Phys. 35 (2012) 530-536

[7] J. A. Aguilar et al., ANTARES Collaboration, Astropart. Phys. 34 (2011) 652-662

[8] A. Klotz, M. Boër, J. L. Atteia and B. Gendre, AJ 137 (2009) 4100-4108

[9] D. M. Coward et al., Publications of the Astron. Soc. of Australia 27 (2010) 331-339

[10] V. Lipunov et al., Astron. Soc. of India Conf. Series 7 (2012) 275

[11] D. N. Burrows et al., Space Sci. Rev. 120 (2005) 165-195

[12] C. W. Akerlof et al., PASP 115 (2003) 132-140

[13] D. J. Schlegel, D. P. Finkbeiner and M. Davis, ApJ 500 (1998) 525-533 